\title{
Transparent, biocompatible nanostructured surfaces for cancer cell capture and culture
}

\author{
This article was published in the following Dove Press journal: \\ International Journal of Nanomedicine \\ 22 May 2014 \\ Number of times this article has been viewed
}

\author{
Boran Chengl,* \\ Zhaobo $\mathrm{He}^{2, *}$ \\ Libo Zhao ${ }^{2, *}$ \\ Yuan Fang' \\ Yuanyuan Chen' \\ Rongxiang $\mathrm{He}^{2}$ \\ Fangfang Chen' \\ Haibin Song' \\ Yuliang Deng ${ }^{2}$ \\ Xingzhong Zhao ${ }^{2}$ \\ Bin Xiong'
}

'Department of Oncology, Zhongnan Hospital of Wuhan University, Hubei Key Laboratory of Tumor Biological Behaviors, Hubei Cancer Clinical Study Center, Wuhan, Hubei, People's Republic of China; ${ }^{2}$ Key Laboratory of Artificial Micro- and Nano-Structures of Ministry of Education, School of Physics and Technology, Wuhan University, Wuhan, Hubei, People's Republic of China

*These authors contributed equally to this work

Correspondence: Bin Xiong Department of Oncology, Zhongnan Hospital of Wuhan University,

Number 169 Donghu Road, Wuchang

District, Wuhan, 43007I, People's

Republic of China

Tel +86 27678 I 3I52

Fax +862767812892

Email binxiong1961@gmail.com

Xingzhong Zhao

Key Laboratory of Artificial Micro- and Nano-Structures of Ministry of Education, School of Physics and Technology, Wuhan University, Luojia Hill Wuchang District,

Wuhan, 43007I, People's Republic

of China

Tel +86 2787642784

Fax +86 2767812892

Email xzzhao@whu.edu.cn

\begin{abstract}
Circulating tumor cells (CTCs) in the blood which have detached from both the primary tumor and any metastases may be considered as a "liquid biopsy" and are expected to replace tumor biopsies in the monitoring of treatment response and determining patient prognosis. Here, we introduce a facile and efficient CTC detection material made of hydroxyapatite/ chitosan (HA/CTS), which is beneficial because of its transparency and excellent biological compatibility. Atomic force microscopy images show that the roughness of the HA/CTS nanofilm (HA/CTSNF) substrates can be controlled by changing the HA:CTS ratio. Enhanced local topographic interactions between nano-components on cancer cell membranes, and the antibody coated nanostructured substrate lead to improved CTC capture and separation. This remarkable nanostructured substrate has the potential for CTC culture in situ and merits further analysis. CTCs captured from artificial blood samples were observed in culture on HA/CTSNF substrates over a period of 14 days by using conventional staining methods (hematoxylin eosin and Wright's stain). We conclude that these substrates are multifunctional materials capable of isolating and culturing CTCs for subsequent studies.
\end{abstract}

Keywords: cell capture, cell culture, nanofilms, hydroxyapatite/chitosan

\section{Introduction}

Solid tumors are the most common cause of morbidity and mortality worldwide ${ }^{1,2}$ due to the lack of effective early detection methods and treatments to prevent metastases. ${ }^{3,4}$ Since the first literature report in 1869, circulating tumor cells (CTCs) ${ }^{5}$ (cancer cells that break away from solid tumors $)^{6}$ have been thought to play a crucial role in tumor metastasis and disease progression. In addition, CTCs considered as a "liquid biopsy" of the tumor, are expected to replace surgical tumor biopsies in the monitoring of treatment response and determining patient prognosis. Detection and characterization of CTCs has been technically challenging however, owing to the extremely low abundance of CTCs (a few to hundreds per milliliter) among $10^{9}$ hematologic cells $\mathrm{mL}^{-1}$ in the blood..$^{7,8}$ In the past decades, a significant amount of research has been devoted to developing CTC detection technology. Because of this, CTC counts in patients' blood can now serve as prognostic indicators and be used in the monitoring of treatment responses in patients with solid tumors. The immunomagnetic separation-based CellSearch ${ }^{\mathrm{TM}}$ Assay (Veridex, Raritan, NJ, USA) is the only US Food and Drug Administration (FDA) cleared CTC technology for the diagnosis of metastatic breast, prostate, and colorectal cancers. Because of its unsatisfactory CTC capture efficiency and high cost, significant efforts have been made to develop new CTC isolation technologies.

A high efficiency CTC detection assay has been reported which uses threedimensional nanostructured substrates based on silicon. ${ }^{9}$ Enhanced local topographic interactions ${ }^{10,11}$ between nano-components on the cancer cell membranes (eg, microvilli 
and filopodia) and antibody-coated nanowires lead to improved CTC capture efficiency. This effect has been further confirmed with other types of nanostructured substrates, including horizontally packed titanium dioxide $\left(\mathrm{TiO}_{2}\right)$ nanofibers ${ }^{12}$ and polymer-grafted silicon nano-substrates. ${ }^{13}$ In most cases, preparation of these nanostructures requires either specialized equipment or complex process control, so the methods have been limited for high throughput fabrication. Further applications of these platforms are also constrained by their nontransparent nature which makes them incompatible with many optical imaging systems such as those using immunocytochemical techniques.

Herein, we introduce a simple but efficient CTC detection material made of hydroxyapatite/chitosan (HA/CTS), which is beneficial due to its transparency and excellent biological compatibility. Owing to their biocompatibility, ${ }^{14-18} \mathrm{HA} / \mathrm{CTS}-$ based composites have been widely used in bioengineering applications such as bone repair, ${ }^{19,20}$ tissue engineering, ${ }^{21}$ and controlled drug release. ${ }^{22}$ However, they have not been used for the development of biomaterials for cancer cell detection. We therefore attempted to reproduce the success with threedimensional nanostructured substrates previously mentioned by using a layer of horizontally oriented HA/CTS composite nanostructures.

\section{Materials and methods}

\section{Preparation and characterization of HA/CTS nanofilm substrates}

A description of the HA/CTS nanofilm (HA/CTSNF) preparation follows. Firstly, the glass was plasma treated: the glass substrate was boiled in piranha solution (3:1 volume/volume $[\mathrm{v} / \mathrm{v}] \mathrm{H}_{2} \mathrm{SO}_{4} / \mathrm{H}_{2} \mathrm{O}_{2}$ ) at $100^{\circ} \mathrm{C}$ for 1 hour, rinsed in deionized (DI) water several times, treated with oxygen plasma (PDC32G, Harrick Plasma, Ithaca, NY, USA) for 2 minutes, and then dried with nitrogen gas for further use. Then, a chitosan aqueous solution of 2 weight percentage (wt $\%$ ) was prepared by dissolving chitosan powder (Haisheng Co, Ltd, Qingdao, People's Republic of China) in distilled water containing $2 \%$ acetic acid, then $5 \mathrm{wt} \%$ nano-hydroxyapatite (HA) powder (Sigma-Aldrich Co., St Louis, MO, USA) was added slowly. The mixed solution was blended with a magnetic stirrer for 12 hours until thoroughly dissolved and then uniformly coated on the clean glass using a photoresist spinner $(6,000 \mathrm{r} / \mathrm{h}, 60 \mathrm{~s})$. In the baking process the coated solution was heated $\left(100^{\circ} \mathrm{C}\right)$ on a bake table for 1 hour. Following the baking process the HA/CTSNF substrate was soaked in $10 \mathrm{wt} \%$ sodium hydroxide $(\mathrm{NaOH})$ solution for 10 hours. Finally, the substrate was washed with DI water, dried, and was now ready for chemical modification. The morphology of the HA/CTSNF substrate was observed by a field emission scanning electron microscope (SEM, 6700F, JEOL, Tokyo, Japan). The surface roughness of the nanofilm substrate was characterized using an atomic force microscope (AFM) (SPM 9500J3; Shimadzu, Kyoto, Japan). The transmittance of the CTCs capture device was measured by an ultravioletvisible (UV-Vis) spectrophotometer (UV-2550; Shimadzu).

\section{Surface modification with streptavidin}

In order to validate the streptavidin (SA) binding to the HA/ CTSNF substrate, we used Cy5-SA (a red fluorescent labeled SA) (Thermo Fisher Scientific, Waltham, MA, USA) as the detector. First, the substrate was modified with $4 \%(\mathrm{v} / \mathrm{v})$ 3-mercaptopropyl trimethoxysilane (Sigma-Aldrich Co.) in ethanol at room temperature for 1 hour. ${ }^{23}$ Then, the substrate was treated with the coupling agent $\mathrm{N}-\mathrm{y}$-maleimidobutyryloxy succinimide ester (GMBS) $0.25 \mathrm{mM}$ (Sigma-Aldrich Co.) for 1 hour make for GMBS attachment to the substrate. Afterwards, the substrate was treated with $50 \mu \mathrm{g} \mathrm{mL}^{-1}$ of Cy5-SA at room temperature for 45 minutes, resulting in the immobilization onto GMBS, and subsequently flushed with $1 \times$ phosphate buffered saline (PBS) to remove excess SA. The fluorescence images (Figure S1) show that the Cy5-SA is binding to the HA/CTSNF substrate. According to the statistics, mean fluorescence intensity of the HA/CTSNF substrate is 5-10 times that of the glass. ${ }^{24}$ Due to the strong and specific biotin-SA interaction, the HA/CTSNF substrate could be modified with biotinylated anti-human EpCAM/TROP1 antibody (goat immunoglobulin G) (anti-EpCAM, R\&D Systems, Minneapolis, MN, USA) for specific cancer cell capture.

\section{Cells}

The colorectal cancer cell line (HCT116), lung cancer cell line (A549), gastric carcinoma cell line (MGC803), cervical cancer cell line (HeLa), and chronic myelogenous leukemia cell line (K562) were harvested from Hubei Key Laboratory of Tumor Biological Behaviors (Hubei, People's Republic of China). Cells were cultured in Dulbecco's Modified Eagle's Medium (DMEM) (BD Biosciences, San Jose, CA, USA) and supplemented with $10 \%$ fetal bovine serum (Sigma-Aldrich Co.) and 1\% penicillin/streptomycin solution at $37^{\circ} \mathrm{C}$ under a humidified $5 \% \mathrm{CO}_{2}$ atmosphere.

\section{Blood samples}

Whole blood samples from healthy donors were obtained from the Department of Clinical Laboratory, Zhongnan Hospital of Wuhan University according to Institutional 
Review Board (IRB) protocol and cancer patients' blood samples were obtained from the Department of Oncology, Zhongnan Hospital of Wuhan University under a separate IRB-approved protocol. All patients gave their informed consent and the research was approved by the Ethics Committee of Zhongnan hospital of Wuhan University. All blood specimens were collected into anticoagulant tubes (EDTA-K ${ }_{2}, 2 \mathrm{~mL}$, violet cap) (Wuhan Zhiyuan Medical Technology Co, Ltd, Wuhan, People's Republic of China).

\section{Cell capture and culture}

Prior to cell capture, a $1 \times 1 \mathrm{~cm}$ substrate was placed into a size-matched chamber after which $15 \mu \mathrm{L}$ of biotinylated anti-EpCAM $\left(10 \mu \mathrm{g} \mathrm{mL}^{-1}\right.$ in PBS with $1 \%[\mathrm{w} / \mathrm{v}]$ bovine serum albumin) was dropped onto the substrate to incubate for 2 hours before washing with PBS. Then, $1 \mathrm{~mL}$ of cell suspensions $\left(10^{5}\right.$ cells $\left.\mathrm{mL}^{-1}\right)$ was loaded. After placing in a cell incubator for 60 minutes under the conditions of $37^{\circ} \mathrm{C}$ and $5 \% \mathrm{CO}^{2}$, the substrate was gently washed with PBS at least eight times. The captured cells on the substrate were fixed with 4\% paraformaldehyde (PFA) in PBS for 10 minutes. Next, the substrate was loaded with $0.5 \mathrm{~mL}$ of $0.2 \%$ Triton X-100 (Sigma-Aldrich) in PBS and incubated for 10 minutes in order to improve the cell permeability and to allow for intracellular staining, followed by staining with 4',6-diamidino-2-phenylindole dihydrochloride (DAPI; $0.33 \mu \mathrm{g} \mathrm{mL}^{-1}$ in DI water) on the target cells for 10 minutes. Finally, we imaged and detected targeted cells using a fluorescence microscope (IX81; Olympus, Tokyo, Japan). CTCs captured on substrates were photographed using IPP software (Media Cybernetics Inc., Silver Spring, MD, USA).

For the cell culture experiment, artificial blood samples were prepared by spiking 1,000 HCT116 cells into $1 \mathrm{~mL}$ healthy donor blood $\left(1,000\right.$ cells $\left.\mathrm{mL}^{-1}\right), 1 \mathrm{~mL}$ suspension was introduced onto anti-EpCAM-coated HA/CTSNF substrate, after incubation for 1 hour, rinsed three times with PBS to remove nonspecific interaction cells (white blood cells [WBCs]), then cultivated in DMEM over a period of 14 days. Normal plate culture was also examined as a control. The medium was replaced once a day.

\section{Scanning electron microscopy morphology observation}

The cell captured sample was prepared following the procedure described in our previous paper, for SEM observation. ${ }^{12}$ Briefly, the cells were fixed with $2 \%$ glutaraldehyde for 2 hours and then dehydrated sequentially with a series of different concentrations of ethanol $(50 \%, 70 \%, 90 \%, 100 \%$, and $100 \%$ ) for 15 minutes followed by further dehydration. After completely dried, the samples were sputter coated with gold before being observed with the field emission SEM (6700F; JEOL) at an acceleration voltage of $20 \mathrm{keV}$.

\section{Cell capture from the artificial CTC blood samples}

As summarized in Table S1, the cell capture efficiency of the optimal capture condition was validated using EpCAMcoated HA/CTSNF substrates to capture target cells (HCT116) from the two kinds of artificial blood sample: 1) $1 \mathrm{~mL}$ DMEM containing HCT116 cells, and 2) $1 \mathrm{~mL}$ human blood (healthy donor) containing HCT116 cells. After rinsing, followed by staining of anti-CK, anti-CD45, and DAPI, the specifically captured HCT116 cells were identified and counted on the substrates.

\section{Results and discussion}

Preparation of the HA/CTSNF substrate is controlled by reaction conditions, including reagent concentrations, temperature, and $\mathrm{pH}$, all of which can affect the morphology of the resultant nanofilms. The structure of the substrate is illustrated in Figure 1. In order to obtain an optimal nanostructured substrate, we tested various reaction conditions for the fabrication. Ultimately, five HA/CTSNF-deposited substrates (HA/CTSNF1 to HA/CTSNF5) were prepared using different HA/CTS compositions, as shown in Figure 2A-E. AFM images show that the square average roughness of the substrates can be controlled by changing the HA:CTS ratio (Figure S2 and Table S2). The HA/CTSNF substrates were formed as described in Materials and methods section. The transmittance of the HA/CTS nanofilm on glass approximately $60 \%$ - is shown in Figure 3A, Figure 3B shows HCT116 cells captured by our HA/CTSNF substrate after 14 days of culture, in which the cell structures can be distinguished via hematoxylin eosin stain and Wright's stain. These remarkable properties allow us to observe the process of detecting cancer cells by microscopy, and potentially obtaining biologically functional cancer cells that can be used for ex vivo culture. In addition, they facilitate downstream analyses of circulating tumor cells from cancer patients by using techniques such as immunocytochemistry, fluorescence in situ hybridization, and genomic analysis. During optimization of the HA/CTSNF substrate for cell capture, two critical parameters were found to be closely related to cell capture efficiency:

1) dimension and roughness of the nanofilms, with capture depending on enhanced local topographic interactions between nano-components on cancer cell membranes 


\section{HA/CTS nanofilms}

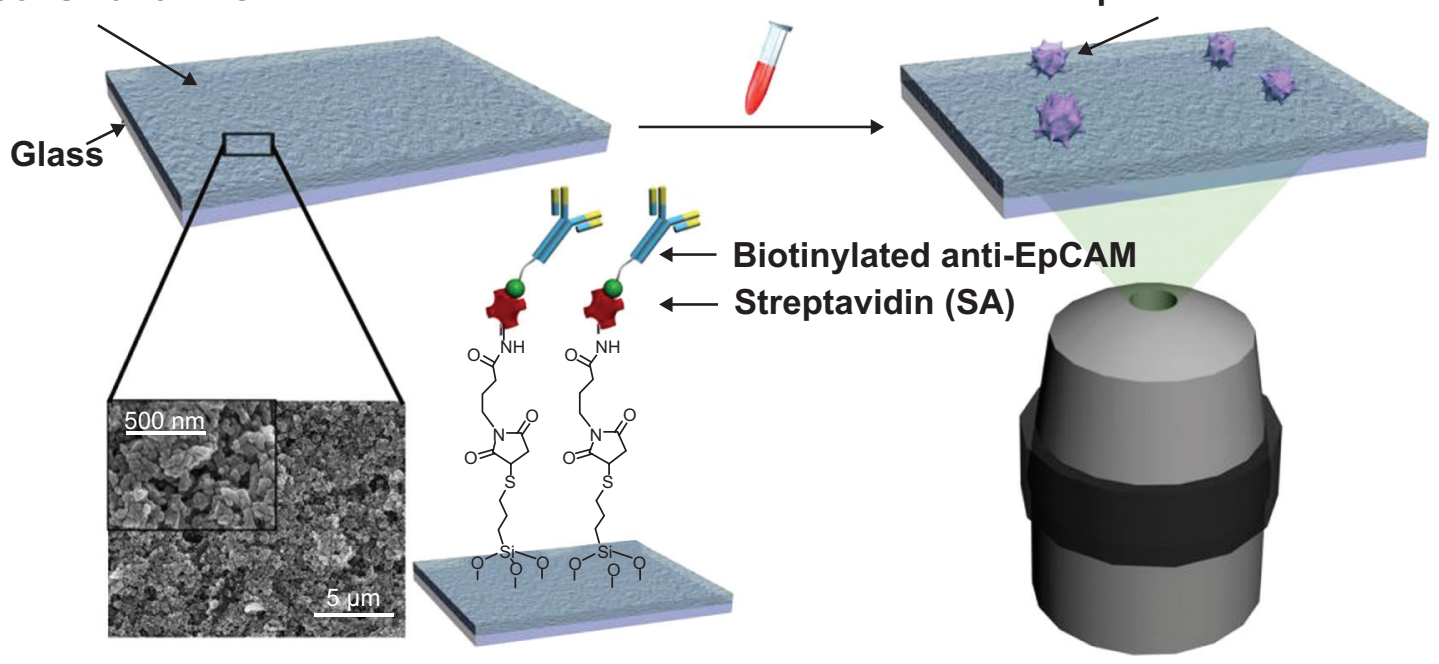

Figure I Horizontally packed HA/CTSNF for improved CTC capture by combining cell capture agent and cancer cell preferred nanoscale topography. Abbreviations: CTC, circulating tumor cell; HA/CTSNF, hydroxyapatite/chitosan nanofilm.

(eg, microvilli and filopodia) and the antibody-coated nanostructured surface. The optimal surface structure allows increased contact frequency and interaction between the cells and the substrate, thus improving capture efficiency.

2) The incubation time, because it takes time, both for the viable cells to approach the substrate and, once contact has been made, for mobility of the cell membrane and extracellular matrix to permit more of the nanostructure to become embedded in the cellular surface components.

To demonstrate how the dimensions and roughness of HA/CTS nanofilm impact the efficiency of cell capture, we prepared an EpCAM-positive cancer cell line (HCT116) in DMEM at a concentration of $10^{5} \mathrm{~mL}^{-1}$ and captured these spiked cells with anti-EpCAM-grafted substrates prepared with composites having different HA:CTS ratios (1.25:2, 2.5:2, 5:2, 10:2, and 15:2 wt \%). As the HA:CTS ratio increased, initially so did the cell capture efficiency, before reaching a maximum, and then declining (Figure $2 \mathrm{G}$ and Table S2). When the roughness of the substrate was approximately 150-200 nm, maximum cell capture numbers were achieved for the HCT116 cell line (approximately 55,000 cells cm ch). $^{-2}$. Presumably, these results indicate that the cellular surface components and the HA/CTSNF are well-matched, allowing sufficient contact and efficient adhesion, resulting in improved cell/substrate affinity. High CTC-capture efficiency was achieved by combining a cell capture agent (anti-EpCAM) with cancer cell-compatible nanoscale topography of the HA/CTSNF substrate. Local topographic interactions correlate with the characteristics of the nano-HA/CTS compos- ite that include substrate roughness, and cell capture yield reaches a maximum at the optimal roughness level. In our test system, this occurred at a roughness of 150-200 nm (HA/CTSNF3, HA/CTS $=5: 2 \mathrm{wt} \%$ ), which was therefore selected for subsequent studies. Next, to explore how film-formation temperature impacts on the efficiency of cell capture, we carried out experiments with the optimal substrates (HA/CTSNF3, HA/CTS $=5: 2 \mathrm{wt} \%$ ) according to the previously described procedure, but used a different baking temperature $\left(40^{\circ} \mathrm{C}, 70^{\circ} \mathrm{C}, 100^{\circ} \mathrm{C}, 130^{\circ} \mathrm{C}\right.$, and $\left.160^{\circ} \mathrm{C}\right)$. There were no observable differences in the numbers of HCT116 cancer cells captured when the baking temperature was increased from $70^{\circ} \mathrm{C}$ to $160^{\circ} \mathrm{C}$, nor was any change observed in the HA/CTSNF surface morphology (Figure S3). It was yet another sign that nanostructured materials could affect the efficiency of the cell capture.

We performed experiments to determine the incubation time required for the most efficient cell capture, using the optimal substrate coated with anti-EpCAM, as previously described. HCT116 cancer cells were spiked into DMEM at a concentration of $10^{5}$ cells $\mathrm{mL}^{-1}$ and captured using our platform. Figure $2 \mathrm{H}$ shows the cell capture yields for different incubation times. For the EpCAM-positive cell line (HCT116), the recovery yields of cell capture increased significantly when increasing the incubation time. The maximal recovery yields were several-fold greater than the minimal yields in our test, with the maximal (saturation) recovery yields being achieved at incubation times of 60 minutes or longer, leading us to adopt 60 minutes as the minimum incubation time for optimal capture. 

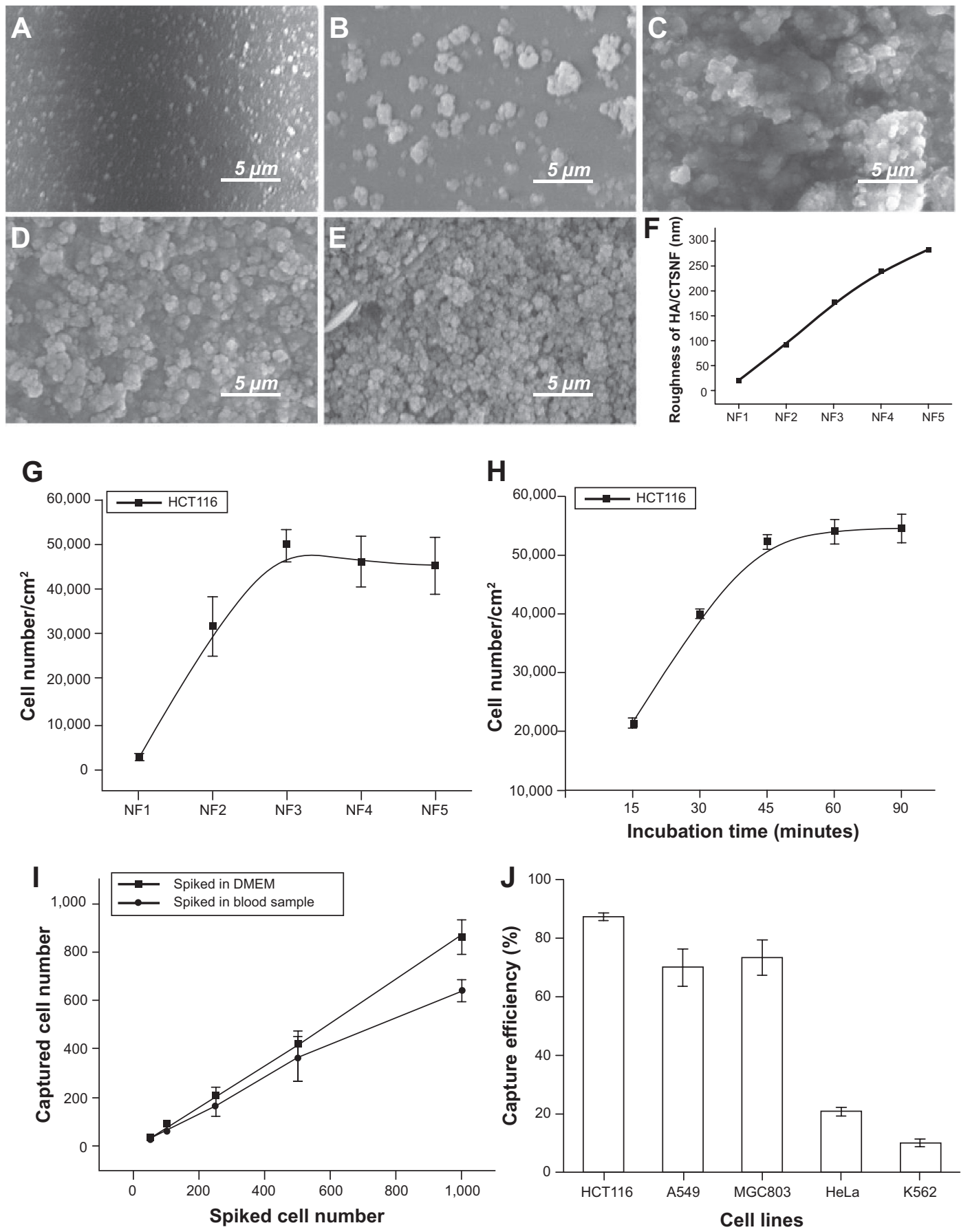

Figure 2 SEM micrograph of HA/CTS nanofilm (HA/CTSNF) substrates with different HA/CTS ratios.

Notes: (A) 1.25:2 wt\% (NFI), (B) 2.5:2 wt\% (NF2), (C) 5:2 wt\% (NF3), (D) 10:2 wt\% (NF4), and (E) I5:2 wt\% (NF5). (F) Plotted square average roughness of different HA/CTSNF samples. The number of cells captured on HA/CTSNF substrate with (G) different samples of NFI, NF2, NF3, NF4 and NF5, and (H) at different incubation times (with incubation time of 15, 30, 45, 60, and 90 minutes). (I) The captured cell number of CTCs was validated from two kinds of artificial blood samples under optimal conditions (HA/CTSNF substrate with HA/CTS, 5:2 wt\% composite and 60 minutes cell incubation time). (J) Cell capture efficiencies on HA/CTSNF substrates were validated using suspensions of EpCAM+ colon (HCTII6), lung (A549), gastric (MGC803), EpCAM- cervical (HeLa), and myelogenous leukemia (K562) cell lines. Error bars show standard deviations $(\mathrm{n}=3)$.

Abbreviations: CTCs, circulating tumor cells; DMEM, Dulbecco's Modified Eagle's Medium; SEM, scanning electron microscope; wt\%, weight percentage; HA/CTS, hydroxyapatite/chitosan.

We validated the cell capture efficiency of the EpCAM-coated HA/CTSNF substrates by using a series of artificial blood samples prepared by spiking HCT116 cells into DMEM and healthy donor's blood at concentrations of approximately $50,100,250,500,1,000$ cells $\mathrm{mL}^{-1}$. The results (Figure 2I and Table S1) indicate that the spiked HCT116 cells were captured from healthy donor's blood with a yield of $64 \%$. These results represent a vast improvement 
A

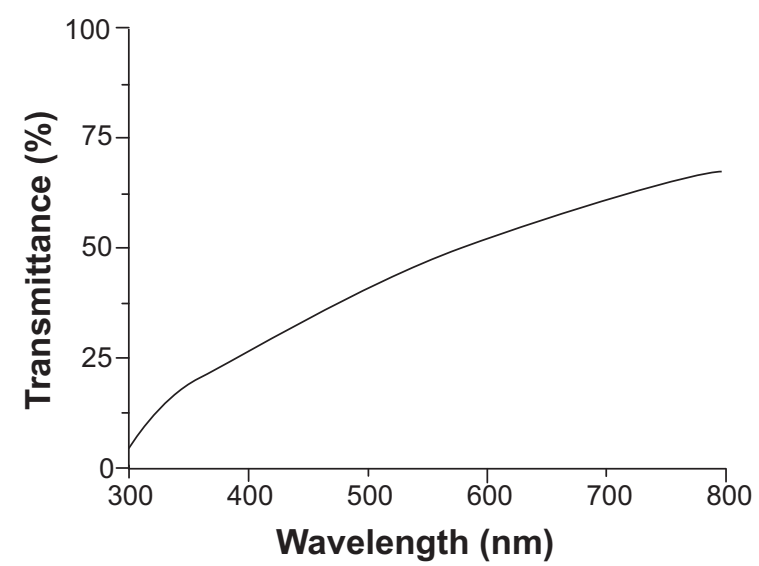

B

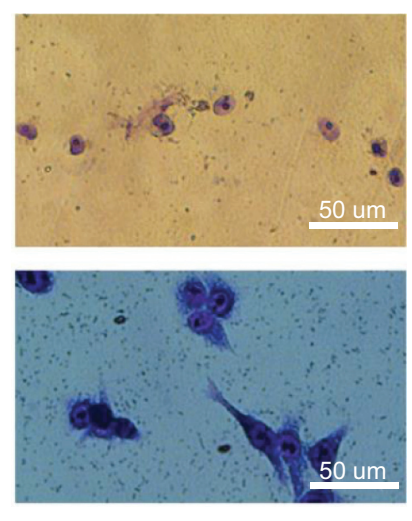

Figure 3 (A) The transmittance of the HA/CTSNF substrate, (B) HE stain (top) and Wright's stain (bottom) of captured HCTII6 cells from whole blood samples. Abbreviations: HA/CTSNF, hydroxyapatite/chitosan nanofilm; HE, hematoxylin eosin.

in capture efficiency compared with those obtained with commercialized technology. ${ }^{25}$

Finally, to illustrate the cell capture specificity and optimized cell capture performance of HA/CTSNF, we used three EpCAM-positive cancer cell lines (colorectal cancer cell line HCT116, lung cancer cell line A549, and gastric carcinoma cell line MGC803), and two EpCAM-negative cancer cell lines (chronic myelogenous leukemia cell line K562 and cervical cancer cell line HeLa) for specific cell capture comparison experiments using the optimized cell capture conditions previously described. For the EpCAM-positive cells, the maximal (ie, saturation) cell capture numbers were achieved, whereas only relatively low numbers of EpCAM-negative cells were captured. The results summarized in Figure 2J show that the EpCAM-coated HA/CTSNF substrates were capable of efficiently capturing HCT116, A549, and MGC803 cells, but not HeLa and K562 cells.

To compare the cell capture performance of the HA/CTSNF substrates with an alternative nanoscale substrate, we prepared an HCT116 cell suspension $\left(10^{5}\right.$ cells $\left.\mathrm{mL}^{-1}\right)$ as previously described. One mL HCT116 cell suspensions prestained with fluorescein diacetate ( $50 \mu \mathrm{g} \mathrm{mL}^{-1}$ in acetone) was introduced onto anti-EpCAM-coated HA/CTSNF substrate (SA-anti-EpCAM-HA/CTSNF) and $\mathrm{TiO}_{2}$ nanofibers substrate (SA-anti-EpCAM-TiNF). A flat glass substrate modified with anti-EpCAM (SA-anti-EpCAM flat glass) acted as a control. After capture, rinsing, and counting, the number of cells captured on HA/CTSNF substrate was equal to that on the horizontally packed $\mathrm{TiO}_{2}$ nanofibers, and much more than that on the flat glass substrate. The results are summarized in Figure 4A. Importantly, no nanoscale components of the cellular surface (microvilli and filopodia) were observed on the cells on the glass substrate, whereas the cells attached to the HA/CTSNF substrate exhibited extended pseudopodia. This finding provides further evidence that the mechanism of our nanostructure-based cell-capture system is a specific surface-to-surface interaction. In addition to high capture efficiency equal to that of our previously described $\mathrm{TiO}_{2}$ nanofibers, cancer cells captured on the HA/CTSNF substrate can be monitored by simultaneous fluorescence and brightfield imaging because of the material's transparent nature. HA/CTSNF is therefore unique in its ability to permit direct monitoring of captured cancer cells. Fluorescein diacetate staining (Figure 4B) shows that the HA/CTSNF-coated area captured considerably more HCT116 cancer cells than the flat glass. To exclude the possibility that HCT116 cells were captured mainly as a result of nonspecific interactions with the HA/CTSNF substrates, we compared the optimized conditions side by side with two control conditions: 1) HA/CTSNF substrate without any surface modification (bare-HA/CTSNF); 2) HA/CTSNF substrate coated only with SA (SAHA/CTSNF), as showed in Figure 4A. The cell capture efficiency of cells captured on both bare-HA/CTSNF and SAHA/CTSNF were around $5 \%$, but exceeded $84 \%$ when the substrate was modified with both SA and the anti-EpCAM capture agent. These data indicate that non-specific cell capture by pure HA/CTSNF and chemically modified HA/CTSNF is negligible. Anti-EpCAM modified HA/CTSNF substrate can capture target cancer cells with high efficiency and specificity.

Next, we assessed the ability of captured cells to be cultured on HA/CTSNF. We prepared artificial blood samples containing $1,000 \mathrm{CTC} \mathrm{mL}^{-1}$ and used $1 \mathrm{~mL}$ for cell capture with anti-EpCAM-coated HA/CTSNF substrate, and cultivated the captured cells in DMEM for 14 days. A normal plate culture was performed in parallel as a control. Morphological observations show (Figure 5A) that 
A

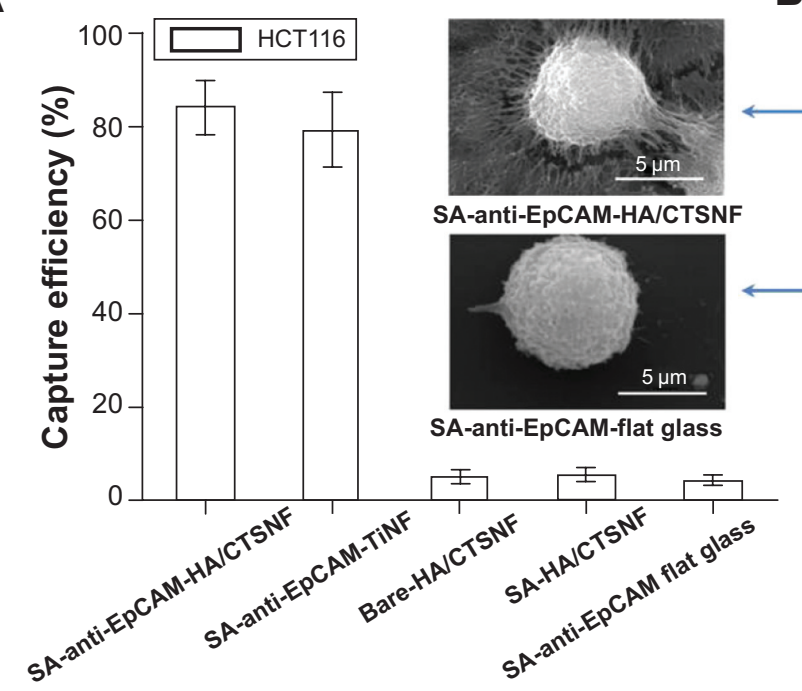

B

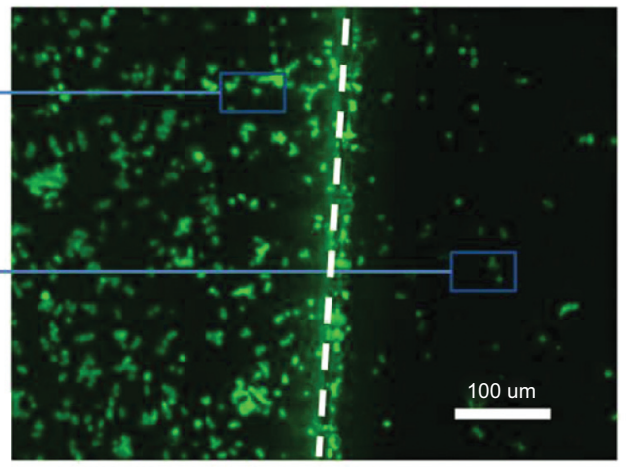

Figure 4 Cell capture performance under five different conditions.

Notes: (A) I) HA/CTSNF substrate modified with anti-EpCAM (SA-anti-EpCAM-HA/CTSNF), 2) TiO ${ }_{2}$ substrate modified with anti-EpCAM (SA-anti-EpCAM-TiNF), 3) HA/CTSNF substrate without any surface modification (bare-HA/CTSNF), 4) HA/CTSNF substrate with only SA coating (SA-HA/CTSNF), and 5) flat glass substrate modified with anti-EPCAM (SA-anti-EpCAM flat glass). An EpCAM-positive cell line (HCTII6) was used. SEM images comparing HCTII6 cell line captured by HA/CTSNF substrate (top) and flat glass substrate (bottom). (B) Fluorescein diacetate fluorescence micrograph of HCTII6 cells captured on HA/CTSNF (left) substrate and glass (right).

Abbreviations: HA/CTSNF, hydroxyapatite/chitosan nanofilm; $\mathrm{TiO}_{2}$, titanium dioxide; SA, streptavidin.

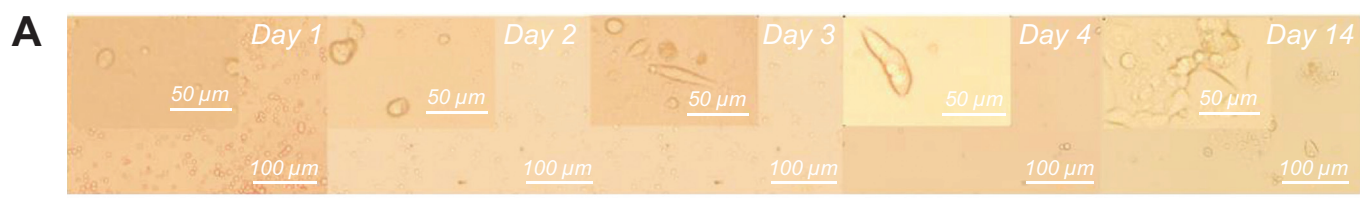

Control-HCT116 culture (plate)

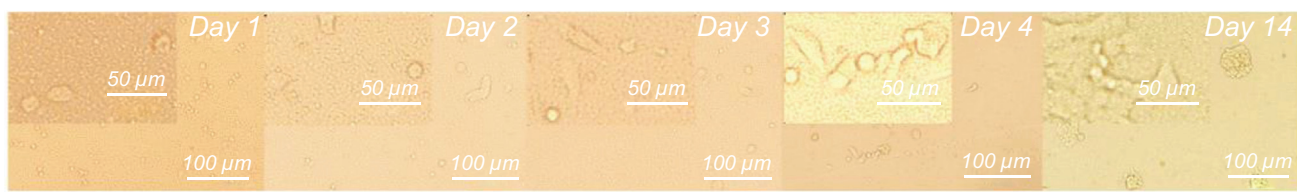

Experiment-captured HCT116 from whole blood samples (HA/CTSNF substrate)

B
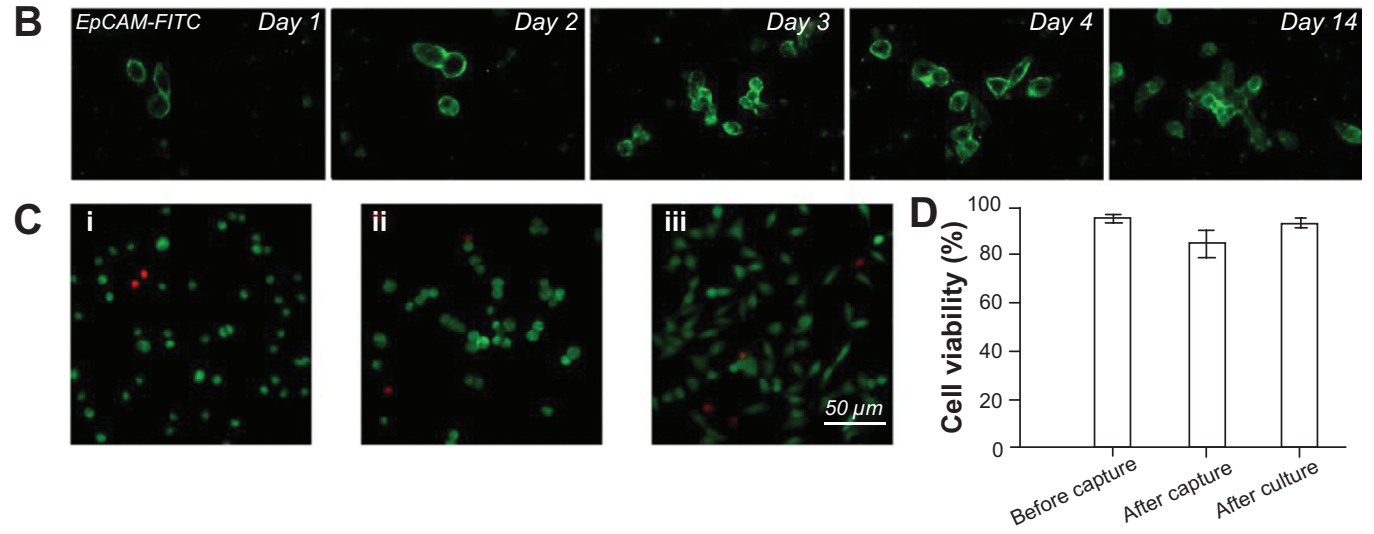

Figure 5 Comparison of cell culture and proliferation between cells.

Notes: (A) Comparison of cell culture and proliferation between cells captured from whole blood samples (I,000 cells $\left.\mathrm{mL}^{-1}\right)$ on anti-EpCAM coated HA/CTSNF substrate and normal plate culture over 14 days. No differences were observed in cell proliferation rates for the HCTI I6 cancer cells. (B) Fluorescent images of captured HCTII 6 cancer cells from whole blood samples ( 1,000 cells $\mathrm{mL}^{-1}$, cultured after capture on anti-EpCAM coated HA/CTSNF substrate). FITC-labeled anti-EpCAM was used. (C) Fluorescent micrographs of fluorescein diacetate-PI stain HCT-II6 cancer cells before capture i); after capture ii); and after culture iii). Viable (green) and non-viable (red) cancer cells are distinguishable. (D) Plotted viability of HCT-II6 cancer cells at different stages. Error bars show standard deviations $(\mathrm{n}=3)$. Abbreviations: HA/CTSNF, hydroxyapatite/chitosan nanofilm; FITC, fluorescein isothiocyanate; PI, propidium iodide. 
the captured HCT116 cells adhered to the substrate, but did not proliferate significantly over the first 3 days, whereas the normal lymphocytes gradually disappeared during the same period. However, after 4 days the cancer cells began to proliferate and migrate on the film, the HA/CTSNF substrate seemed to be more effective for cell adhesion in the initial period of cell culture (up to day 4) because of the good biocompatibility of the nanofilms and their uniform local surface topography. After 14 days, the cell population increased significantly. Figure 5B shows the fluorescein isothiocyanate (FITC) stained HCT116 cancer cells (1,000 cells $\left.\mathrm{mL}^{-1}\right)$ captured from whole blood samples using anti-EpCAM coated HA/CTSNF substrate and cultured over 14 days. Further, cell viability was tested by fluorescein diacetate-propidium iodide, assay at three stages: before capture, after capture, and after culture. The results indicate that cell viability reached $84 \%$ after capture, and $93 \%$ after culture, making these cells suitable for subsequent studies (Figures 5C and 5D). The HA/CTSNF substrate clearly appears to have no negative effects on cell morphology, viability or proliferation, exhibits excellent biocompatibility compared with the regular culture plate, and is suitable for cell culture and real time observation, offering an alternate way to obtain more highly purified CTCs. In addition, because the captured cancer cells are alive, they could be isolated from cancer patients and potentially expanded ex vivo for further molecular interrogation. The transparency of HA/ CTSNF allows direct observation and accurate positioning of the cancer cells it captures, which means they could also potentially be physically isolated for proteomic and genomic analyses.

To validate the clinical utility of the EpCAM-coated HA/CTSNF substrate, we applied the optimized cell capture conditions to study peripheral blood samples from gastrointestinal and lung cancer patients. One $\mathrm{mL}$ peripheral blood sample (preserved in EDTA-K $\mathrm{K}_{2}$ tubes) were introduced onto an EpCAM-coated HA/CTSNF substrate and placed into a size-matched chamber $(1 \times 1 \mathrm{~cm})$. After incubation, the captured cells were fixed with 4\% PFA and permeabilized with $0.02 \%$ Triton-X100. Captured cells were then stained with PE-labeled anti-CK, FITC-labeled anti-CD45 and DAPI. ${ }^{26-29}$ Fluorescence microscopy was used to distinguish specifically captured CTCs (CK+/CD45-/DAPI+) from non-specifically immobilized WBCs (CK-/CD45+/DAPI+) by their fluorescence and larger size (CTCs: 10-30 $\mu \mathrm{m}$; $1 \mathrm{WBCs},<15 \mu \mathrm{m}$ ) (Figure 6A). CTC enumeration results are summarized in Figure 6B and Table 1. CTCs were captured from the peripheral blood of the cancer patients with

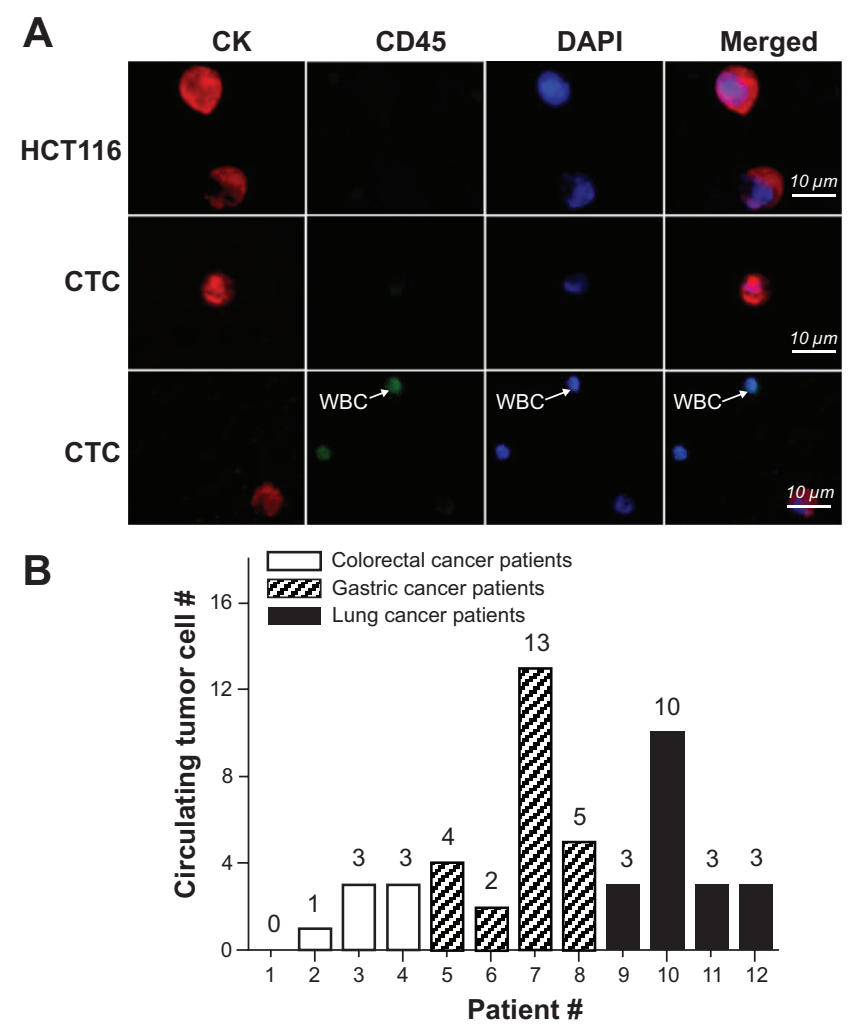

Figure 6 (A) Fluorescent micrographs of CTCs captured from whole blood samples collected from cancer patients. The three-color immunocytochemistry method was used to identify and enumerate CTCs on anti-EpCAM-grafted HA/CTSNF substrate from non-specifically captured WBCs, including FITC-labeled anti-CD45 and PElabeled anti-CK as well as DAPI nuclear staining. The combined information was used to identify CTCs from WBCs. (B) CTC enumeration results obtained from cancer patients (colorectal cancer, gastric cancer and lung cancer).

Abbreviations: CTCs, circulating tumor cells; HA/CTSNF, hydroxyapatite/chitosan nanofilm; FITC, fluorescein isothiocyanate; DAPI, 4',6-diamidino-2-phenylindole dihydrochloride; WBCs, white blood cells.

counts ranging from $0-13$ per $\mathrm{mL}$. The anti-EpCAM modified HA/CTSNF substrate therefore exhibited considerable CTC capture efficiency.

\section{Conclusion}

We have achieved precise control of surface roughness in an HA/CTSNF deposited onto a glass slide to achieve enhanced topographic interactions with CTC surface components. The epithelial-specific capture agent, antiEpCAM, ${ }^{30}$ was conjugated onto the surface of the nanosubstrate to enable specific capture of CTCs. HA/CTSNF thus constructed exhibits CTC capture performance equal to that of horizontally packed $\mathrm{TiO}_{2}$ nanofibers, as well as excellent biocompatibility. In addition, the transparent nature of the HA/CTSNF makes direct observation of the cells possible. These advantageous characteristics enable CTC culture in situ and traditional dyeing, as well as accurate positioning of the immobilized cells for subsequent isolation and purification. 
Table I Cancer patient CTC counts observed on HA/CTSNF substrates, standard TNM cancer staging; metastatic versus localized

\begin{tabular}{|c|c|c|c|c|}
\hline Cancer species & Patient & CTC count (sample $=I \mathrm{~mL}$ ) & TNM & Metastatic \\
\hline \multirow[t]{4}{*}{ Colorectal cancer } & 1 & 0 & T3N2M0 & - \\
\hline & 2 & 1 & T3NOMO & - \\
\hline & 3 & 3 & T4NOMO & - \\
\hline & 4 & 3 & T2NOMO & - \\
\hline \multirow[t]{4}{*}{ Gastric cancer } & 5 & 4 & T4N3M0 & - \\
\hline & 6 & 2 & TINOMO & - \\
\hline & 7 & 13 & T4N2MI & + \\
\hline & 8 & 5 & T4NXMI & + \\
\hline \multirow[t]{4}{*}{ Lung cancer } & 9 & 3 & TINOMO & - \\
\hline & 10 & 10 & TINOMO & - \\
\hline & 11 & 3 & TINIMO & - \\
\hline & 12 & 3 & $\mathrm{~T} 2 \mathrm{bN} \times \mathrm{MO}$ & - \\
\hline
\end{tabular}

Abbreviations: CTC, circulating tumor cell; HA/CTSNF, hydroxyapatite/chitosan nanofilm; TNM, TNM Classification of Malignant Tumours.

CTCs captured from artificial blood samples could be cultured and expanded on HA/CTSNF substrates that represent a multifunctional material capable of CTC isolation and culture, and permitting their immunocytochemical and molecular analysis for clinical applications (monitoring treatment responses and indicating patient prognosis). We anticipate this platform will also be applied to the capture and in situ culture of CTCs for studying drug resistance mechanisms, tumor heterogeneity, and tumor evolution.

\section{Acknowledgment}

The work was supported by the National High Technology Research and Development Program of China (grant number 2012AA02A502, 2012AA02A506).

\section{Disclosure}

The authors report no conflicts of interest in this work.

\section{References}

1. Jemal A, Siegel R, Ward E, et al. Cancer statistics, 2008. CA Cancer J Clin. 2008;58(2):71-96.

2. Marrett LD, De P, Airia P, Dryer D. Cancer in Canada in 2008. CMAJ. 2008;179(11):1163-1170.

3. Pantel K, Brakenhoff RH. Dissecting the metastatic cascade. Nat Rev Cancer. 2004;4(6):448-456.

4. Chambers AF, Groom AC, MacDonald IC. Dissemination and growth of cancer cells in metastatic sites. Nat Rev Cancer. 2002;2:563-572.

5. Ashworth T. A case of cancer in which cells similar to those in the tumours were seen in the blood after death. Aust Med J. 1869;14(3):146-149.

6. Steeg PS. Tumor metastasis: mechanistic insights and clinical challenges. Nat Med. 2006;12(8):895-904.

7. Racila E, Euhus D, Weiss AJ, et al. Detection and characterization of carcinoma cells in the blood. Proc Natl Acad Sci U S A. 1998;95(8): 4589-4594.

8. Zieglschmid V, Hollmann C, Bocher O. Detection of disseminated tumor cells in peripheral blood. Crit Rev Clin Lab Sci. 2005;42(2):155-196.

9. Wang S, Wang H, Jiao J, et al. Three-dimensional nanostructured substrates toward efficient capture of circulating tumor cells. Angew Chemie Int Ed Engl. 2009;48(47):8970-8973.
10. Fischer KE, Alemán BJ, Tao SL, et al. Biomimetic nanowire coatings for next generation adhesive drug delivery systems. Nano Lett. 2009;9(2):716-720.

11. Curtis A, Varde M. Control of cell behavior: topological factors. J Natl Cancer Inst. 1964;33:15-26.

12. Zhang N, Deng Y, Tai Q, et al. Electrospun TiO2 nanofiber-based cell capture assay for detecting circulating tumor cells from colorectal and gastric cancer patients. Adv Mater. 2012;24(20):2756-2760.

13. Sekine J, Luo S, Wang S, Zhu B, Tseng H, Yu H. Functionalized conducting polymer nanodots for enhanced cell capturing: the synergistic effect of capture agents and nanostructures. Adv Mate. 2011;23(41): $4788-4792$.

14. Gomez-Vega JM, Saiz E, Tomsia AP, Marshall GW, Marshall SJ. Bioactive glass coatings with hydroxyapatite and Bioglass particles on Ti-based implants. 1. Processing. Biomaterials. 2000;21(2): 105-111.

15. Yamaguchi I, Tokuchi K, Fukuzaki H, et al. Preparation and microstructure analysis of chitosan/hydroxyapatite nanocomposites. J Biomed Mater Res. 2001;55(1):20-27.

16. Hu Q, Li B, Wang M, Shen J. Preparation and characterization of biodegradable chitosan/hydroxyapatite nanocomposite rods via in situ hybridization: a potential material as internal fixation of bone fracture. Biomaterials. 2004;25(5):779-785.

17. Rusu VM, Ng CH, Wilke M, Tiersch B, Fratzl P, Peter MG. Size-controlled hydroxyapatite nanoparticles as self-organized organic-inorganic composite materials. Biomaterials. 2005;26(26):5414-5426.

18. Doremus RH. Bioceramics. Journal of Materials Science. 1992;27(2): 285-297.

19. Menon P, Mukherjee D. Development of a composite of hydroxylapatite and chitosan as a bone graft substitute. Biomedical Engineering Conference, 1995, Proceedings of the 1995 Fourteenth Southern; 1995: 95-97.

20. Spence ML, McCord MG. A novel composite for bone replacement. Biomedical Engineering Conference, 1997, Proceedings of the 1997. 1997:257-259.

21. Zhang Y, Zhang M. Synthesis and characterization of macroporous chitosan/calcium phosphate composite scaffolds for tissue engineering. J Biomed Mater Res. 2001;55(3):304-312.

22. Zhang Y, Zhang M. Calcium phosphate/chitosan composite scaffolds for controlled in vitro antibiotic drug release. J Biomed Mater Res. 2002; 62(3):378-386.

23. Dupraz AM, De Wijn JR, v d Meer SA, De Groot K. Characterization of silane-treated hydroxyapatite powders for use as filler in biodegradable composites. J Biomed Mater Res. 1996;30(2):231-238.

24. Yin S, Wu YL, Hu B, et al. Three-Dimensional Graphene Composite Macroscopic Structures for Capture of Cancer Cells. Advanced Materials Interfaces. 2014;1(1):1300043. 
25. Allard WJ, Matera J, Miller MC, et al. Tumor cells circulate in the peripheral blood of all major carcinomas but not in healthy subjects or patients with nonmalignant diseases. Clin Cancer Res. 2004;10(20): 6897-6904.

26. Liu H, Liu X, Meng J, et al. Hydrophobic interaction-mediated capture and release of cancer cells on thermoresponsive nanostructured surfaces. Adv Mater. 2013;25(6):922-927.

27. Zhang $\mathrm{P}$, Chen $\mathrm{L}, \mathrm{Xu} \mathrm{T}$, et al. Programmable fractal nanostructured interfaces for specific recognition and electrochemical release of cancer cells. Adv Mater. 2013;25(26):7603-7609.
28. He R, Zhao L, Liu Y, et al. Biocompatible TiO2 nanoparticle-based cell immunoassay for circulating tumor cells capture and identification from cancer patients. Biome Microdevices. 2013;15(4):617-626.

29. Yang G, Liu H, Liu X, et al. Underwater-transparent nanodendritic coatings for directly monitoring cancer cells. Adv Healthc Mater. 2014;3(3):460.

30. Went PT, Lugli A, Meier S, et al. Frequent EpCam protein expression in human carcinomas. Hum Pathol. 2004;35(1):122-128. 


\section{Supplementary materials}

A
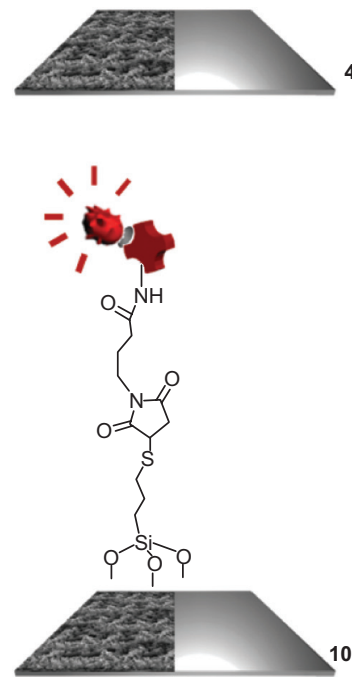

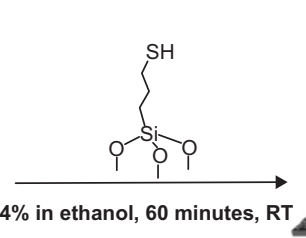

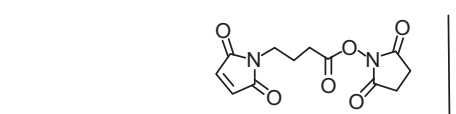

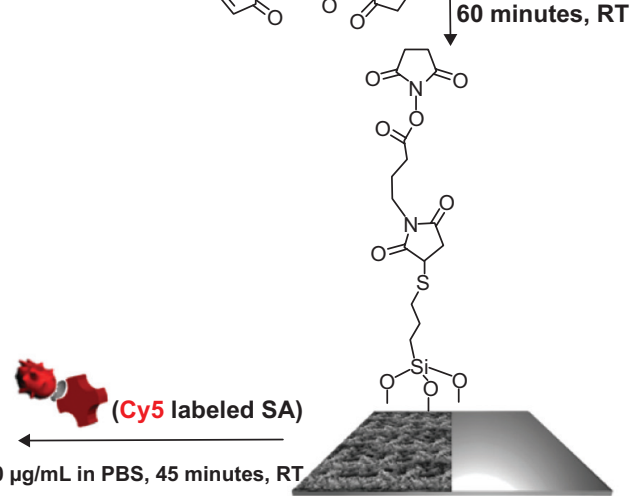

Figure SI Surface modification with streptavidin (SA). (A) Surface modification procedure of the hydroxyapatite/chitosan nanofilm (HA/CTSNF) substrate with SA. (B) Fluorescence micrograph of HA/CTSNF substrate (left) and glass (right) coated with Cy5-SA (red).

Abbreviations: DMSO, dimethyl sulfoxide; RT, room temperature; PBS, phosphate buffered saline.

\section{Capture efficiencies of the EpCAM-coated HA/CTSNF substrates}

Table SI Cell capture efficiencies of the optimal capture conditions (HA/CTSNF substrate with HA/CTS 5:2 weight \% composite, baking temperature of $100^{\circ} \mathrm{C}$, and 60 minutes cell incubation time) were validated using the HA/CTSNF substrates

\begin{tabular}{|c|c|c|c|}
\hline \multirow[t]{2}{*}{ Cell number/mL } & \multicolumn{2}{|l|}{ Capture yield } & \multirow{2}{*}{$\begin{array}{l}\text { Successful detections } \\
\text { out of total trials }\end{array}$} \\
\hline & $\begin{array}{l}\text { I mL DMEM } \\
\text { containing HCT I I } 6 \text { cells }\end{array}$ & $\begin{array}{l}\text { I mL human blood } \\
\text { containing HCT I } 16 \text { cells }\end{array}$ & \\
\hline 50 & $85 \pm 12 \%$ & $67 \pm 21 \%$ & $3 / 3$ \\
\hline 100 & $88 \pm 20 \%$ & $66 \pm 11 \%$ & $3 / 3$ \\
\hline 250 & $84 \pm 13 \%$ & $66 \pm 15 \%$ & $3 / 3$ \\
\hline 500 & $85 \pm 10 \%$ & $72 \pm 18 \%$ & $3 / 3$ \\
\hline 1,000 & $86 \pm 7 \%$ & $64 \pm 4 \%$ & $3 / 3$ \\
\hline
\end{tabular}

Abbreviations: DMEM, Dulbecco's Modified Eagle's Medium; HA/CTSNF, hydroxyapatite/chitosan nanofilm.

HA/CTSNF1
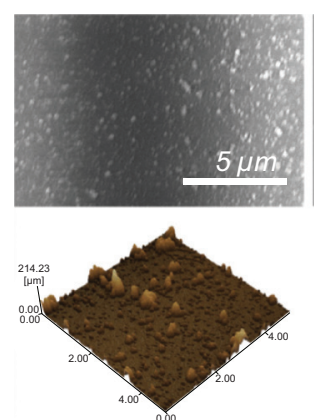

HA/CTSNF2
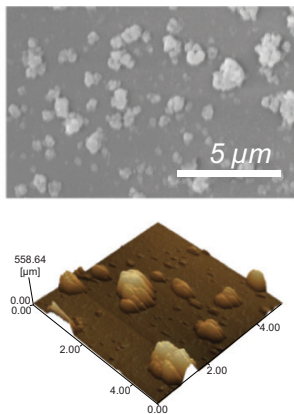

HA/CTSNF3
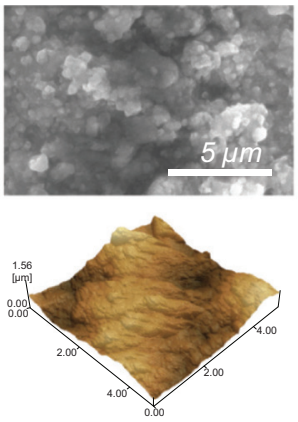

HA/CTSNF4
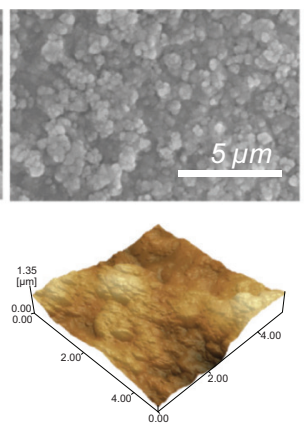

HA/CTSNF5
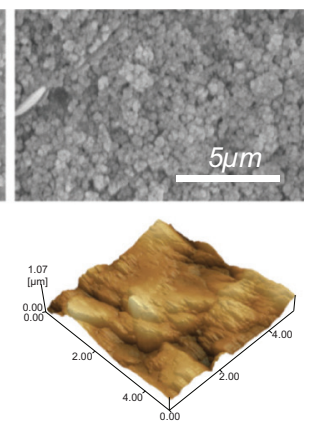

Figure S2 SEM and AFM images of HA/CTSNF substrates with different HA/CTS ratios.

Abbreviations: SEM, scanning electron microscopy; AFM, atomic force microscopy; HA/CTSNF, hydroxyapatite/chitosan nanofilm. 


\section{Preparation and characterization of hydroxyapatite/chitosan (HA/CTS) nanofilm substrates}

Table S2 Concentrations, roughness, and captured cell numbers of HA/CTS nanofilms (HA/CTSNFI to HA/CTSNF5)

\begin{tabular}{llll}
\hline Sample & $\begin{array}{l}\text { Composites } \\
\text { (weight } \%)\end{array}$ & $\begin{array}{l}\text { Square average } \\
\text { roughness }(\mathbf{n m})\end{array}$ & $\begin{array}{l}\text { Captured cell } \\
\text { numbers/cm }\end{array}$ \\
\hline HA/CTSNFI & HA/CTS I.25:2 & 2,833 \\
HA/CTSNF2 & HA/CTS 2.5:2 & 20.136 & 31,905 \\
HA/CTSNF3 & HA/CTS 5:2 & 90.863 & 49,960 \\
HA/CTSNF4 & HA/CTS I0:2 & 176.826 & 46,200 \\
HA/CTSNF5 & HA/CTS I5:2 & 238.883 & 45,366 \\
\hline
\end{tabular}

Abbreviation: HA/CTS, hydroxyapatite/chitosan.
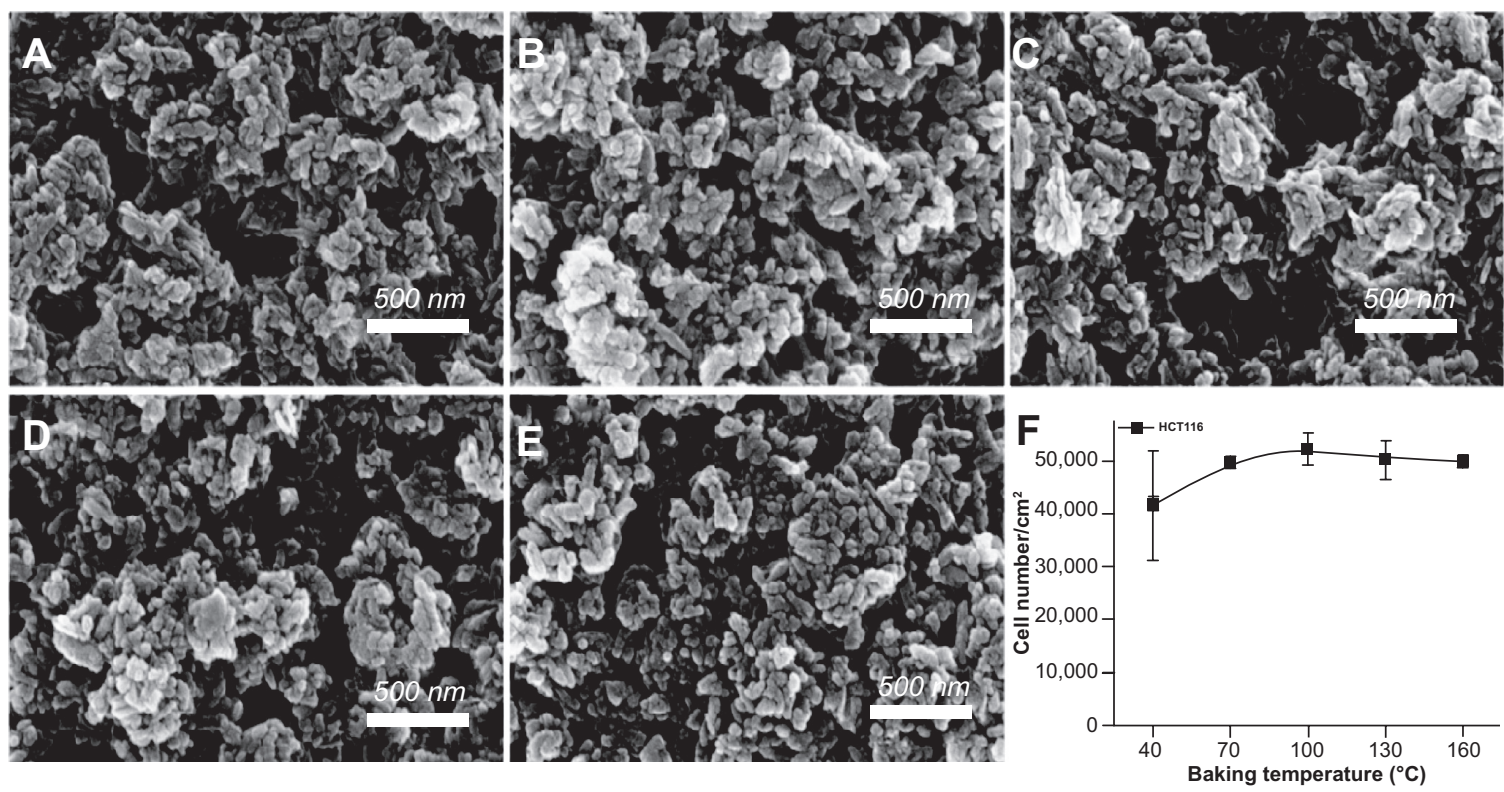

Figure S3 SEM images of HA/CTSNF substrates with different baking temperatures.

Notes: (A) $40^{\circ} \mathrm{C}$, (B) $70^{\circ} \mathrm{C}$, (C) $100^{\circ} \mathrm{C}$, (D) $130^{\circ} \mathrm{C}$, and (E) $160^{\circ} \mathrm{C}$. (F) Plotted number of cells captured on HA/CTSNF substrates with different baking temperatures. No differences were observed in captured cell numbers when the baking temperature was higher than $70^{\circ} \mathrm{C}$ for $\mathrm{HCT}-\mathrm{II} 6$ cancer cells.

Abbreviations: SEM, scanning electron microscopy; HA/CTSNF, hydroxyapatite/chitosan nanofilm.

\section{Publish your work in this journal}

The International Journal of Nanomedicine is an international, peerreviewed journal focusing on the application of nanotechnology in diagnostics, therapeutics, and drug delivery systems throughout the biomedical field. This journal is indexed on PubMed Central, MedLine, CAS, SciSearch ${ }^{\circledR}$, Current Contents $® /$ Clinical Medicine,
Journal Citation Reports/Science Edition, EMBase, Scopus and the Elsevier Bibliographic databases. The manuscript management system is completely online and includes a very quick and fair peer-review system, which is all easy to use. Visit http://www.dovepress.com/ testimonials.php to read real quotes from published authors. 